\title{
Netherlands Ophthalmological Society
}

130th Meeting, at the Wilhelmina-Gasthuis, Amsterdam. December 12th and 13th, 1953

Chairman: Prof. H. M. Dekking. Secretaries: Dr. T. A. Vos and Dr. C. H. O. M. von Winning.

List of Communications.

Crone, R. A.: An Apparatus for the Investigation of Chromatic Sense . 54

Hagedoorn, A.: The State Committee on Orthoptic Training .... 54

Velzeboer, C. M. J.: Orthoptic Training. (Manuscript not received.) .

Discussion: Jonkers, Reiner.

Ten Doesschate, G.: Results of an Investigation on Depth Perception at a Distance of 50 Metres. (Will be published in extenso elsewhere.) . 56 Discussion: Dekking. Plant en, J. Th.: Do Intra-Arterial Obstructive Mechanisms Exist in the Eye? $\quad 57$

Discussion: Ten Thije, Copper, Hagedoorn, Bouws, Planten (reply).

VandenHooff, A.: Electron-Microscopical Structure of Some Ophthalmic

Fibrous Structures 60

Discussion: Van Walbeek, VandenHooff (reply).

De Rooy, A. J. M.: Some Observations on Sutures During, and the Behaviour of Vitreous Humour After Cataract Operations .... 62 Discussion: Flieringa, Mulock Houwer, Dekking, Hagedoorn, De Rooy (reply).

Van Driest, E.: Acute Blindness Followed by Tapetoretinal Degeneration.

(Manuscript not received.) 63

Discussion: Flieringa.

Winkelman, J. E.: Marcus-Gunn's Syndrome

Discussion: Halbertsma, Winkelman (reply).

- Corneal Transplantation in Cornea Guttata 66

Halbertsma, K. T. A.: Epithelioma of the Tarsal Conjunctiva ... 68 Discussion: Dekking.

Hagedoorn, A.: Demonstrations

69

Discussion: Halbertsma, Roster, Rrouwels, Ten Thije, Van Walbeek, Branbergen, Flieringa, Hagedoorn (reply).

Winkelman, J. E., and G. P. M. Horsten: Autokinetic Movements. (Manuscript not received.) 76

Discussion: Pannevis, Zeeman, Dekking, Winkelman (reply).

Bleeker, G. M.: Considerations on Antibiotics in Ophthalmology. (Will be

p. published in full in this Journal.) 76

Discussion: Rok-Van Alphen

Pannevis

Crone

Flieringa

Bleeker (reply). 